\title{
Integration of Gene Expression Profile Data to Screen and Verify Hub Genes Involved in Osteoarthritis
}

\author{
Zhaoyan Li $\mathbb{D}^{1},{ }^{1,2,3}$ Qingyu Wang $\mathbb{D}^{1,1,2,3}$ Gaoyang Chen $\mathbb{D}^{1,},{ }^{1,2,3}$ Xin Li $\mathbb{D}^{1},{ }^{1}$ Qiwei Yang $\mathbb{D}^{\mathbb{D}},{ }^{2,3}$ \\ Zhenwu Du $\mathbb{D}^{1,2,3}$ Ming Ren $\mathbb{D}^{1,3}$ Yang Song $\mathbb{D}^{1,3}$ and Guizhen Zhang $\mathbb{D}^{1,2,3}$ \\ ${ }^{1}$ Department of Orthopedics of the Second Hospital of Jilin University, Ziqiang Street 218, Changchun, Jilin 130041, China \\ ${ }^{2}$ Research Centre of the Second Hospital of Jilin University, Ziqiang Street 218, Changchun, Jilin 130041, China \\ ${ }^{3}$ The Engineering Research Centre of Molecular Diagnosis and Cell Treatment for Metabolic Bone Diseases of Jilin Province, \\ Ziqiang Street 218, Changchun, Jilin 130041, China
}

Correspondence should be addressed to Yang Song; songyangjlu@jlu.edu.cn and Guizhen Zhang; zhangguizhenjlu@163.com

Received 25 June 2018; Revised 30 July 2018; Accepted 5 August 2018; Published 14 August 2018

Academic Editor: Hesham H. Ali

Copyright (c) 2018 Zhaoyan Li et al. This is an open access article distributed under the Creative Commons Attribution License, which permits unrestricted use, distribution, and reproduction in any medium, provided the original work is properly cited.

\begin{abstract}
Osteoarthritis $(\mathrm{OA})$ is one of the most common diseases worldwide, but the pathogenic genes and pathways are largely unclear. The aim of this study was to screen and verify hub genes involved in OA and explore potential molecular mechanisms. The expression profiles of GSE12021 and GSE55235 were downloaded from the Gene Expression Omnibus (GEO) database, which contained 39 samples, including 20 osteoarthritis synovial membranes and 19 matched normal synovial membranes. The raw data were integrated to obtain differentially expressed genes (DEGs) and were deeply analyzed by bioinformatics methods. The Gene Ontology (GO) and pathway enrichment of DEGs were performed by DAVID and Kyoto Encyclopedia of Genes and Genomes (KEGG) online analyses, respectively. The protein-protein interaction (PPI) networks of the DEGs were constructed based on data from the STRING database. The top 10 hub genes VEGFA, IL6, JUN, IL1 $\beta$, MYC, IL4, PTGS2, ATF3, EGR1, and DUSP1 were identified from the PPI network. Module analysis revealed that OA was associated with significant pathways including TNF signaling pathway, cytokine-cytokine receptor interaction, and osteoclast differentiation. The qRT-PCR result showed that the expression level of IL6, VEGFA, JUN, IL-1 $\beta$, and ATF3 was significantly increased in OA samples $(\mathrm{p}<0.05)$, and these candidate genes could be used as potential diagnostic biomarkers and therapeutic targets of OA.
\end{abstract}

\section{Introduction}

Osteoarthritis is a chronic joint disease characterized by degeneration of cartilage, synovial inflammation osteophytes formation, and subchondral bone sclerosis. Its typical signs and symptoms include pain, swelling, and stiffness, often accompanied by a decrease in function and limitation of movement [1]. It is a slowly progressive, disabling joint disorder that significantly reduces the quality of life. By 2030, it is predicted that 67 million people in the United States will be diagnosed with $\mathrm{OA}$ [2]. Although there are extensive studies on the mechanism and etiology in OA formation and progression, the causes of OA are still not clear.

Epidemiological studies have demonstrated that $\mathrm{OA}$ is a complex polygenic disorder with numerous environmental and genetic risk factors, in which one of the contributing factors to disease progression is a genetic component [3]. Over the last 15 years, researches have focused on the search for susceptible sites of osteoarthritis. Genomewide association studies (GWAS) could discover potential genetic variants that could be used as biomarkers for early diagnosis and targeted therapy [4]. At present, with the development of high-throughput sequencing technology, a large number of studies have been performed on osteoarthritis gene expression profiles and screened thousands of differentially expressed genes. However, the results for the expressed mRNAs are inconsistent with different gene profile due to sample heterogeneity or different sequencing platform. Thus, no reliable results have been identified in OA. However, the integrated bioinformatics methods will solve the disadvantages and identify the hub genes involved in OA. 
TABle 1: The primers of top 10 hub genes.

\begin{tabular}{lrr}
\hline Gene name & Forward primer & Reverse primer \\
\hline VEGFA & GTGAATGCAGACCAAAGAAAGA & AGGCTCCAGGGCATTAGAC \\
IL6 & TCAATATTAGAGTCTCAACCCCCA & GAAGGCGCTTGTGGAGAAGG \\
JUN & GAGCTGGAGCGCCTGATAAT & CCCTCCTGCTCATCTGTCAC \\
IL1B & TGAGCTCGCCAGTGAAATGA & AGGAGCACTTCATCTGTTTAGGG \\
MYC & TACAACACCCGAGCAAGGAC & GAGGCTGCTGGTTTTCCACT \\
IL4 & CATCTTTGCTGCCTCCAAGAACA & GTTCCTGTCGAGCCGTTTCA \\
PTGS2 & GCTGTTCCCACCCATGTCAA & AAATTCCGGTGTTGAGCAGT \\
ATF3 & GAGGTGGGGTTAGCTTCAGT & TCATTTTGATTTTGGGGCAAGGT \\
EGR1 & CACCTGACCGCAGAGTCTTT & GAGTGGTTTGGCTGGGGTAA \\
DUSP1 & CTCAAAGGAGGATACGAAGCGTT & CCCTGATCGTAGAGTGGGGT \\
\hline
\end{tabular}

In this work, we have downloaded two microarray datasets GSE12021 [5] and GSE55235 [6] and screened out differentially expressed genes (DEGs) between synovial membranes of knee OA patients and normal controls. GO and pathways enrichment analyses of DEGs were applied and functional module analysis of the protein-protein interaction (PPI) network was also constructed. The study aimed to identify hub genes and explore the intrinsic molecular mechanisms involved in OA.

\section{Materials and Methods}

2.1. Microarray Data Information. The Gene Expression Omnibus (GEO, http://www.ncbi.nlm.nih.gov/geo) is a public genomics data repository which stores gene expression datasets and original series and platform records [7]. The gene expression profiles of GSE12021 and GSE55235 were downloaded from the GEO database which all based on GPL96 (Affymetrix Human Genome U133A Array) platform. The microarray data of GSE12021 include 10 knee osteoarthritis synovial membranes and 9 normal controls, and the microarray data of GSE55235 include 10 knee osteoarthritis synovial membranes and 10 normal controls.

2.2. Data Processing and Identification of DEGs. The process of data preprocessing included background adjustment, normalization, and summarization. The raw data were preprocessed by affy package [8] in $\mathrm{R}$ software and limma package [9] in R software was used to identify the upregulated and downregulated DEGs between osteoarthritis synovial membranes and normal controls. $\mathrm{P}$ values were adjusted using the Benjamini and Hochberg test, and $\mathrm{p}<0.05$ and $|\log \mathrm{FC}|>1$ were considered as the cutoff criterion.

2.3. Gene Ontology (GO) and Pathway Enrichment Analyses. DAVID (the Database for Annotation, Visualization, and Integrated Discovery) online bioinformatics database integrates biological data and analysis tools to provide systematic annotation information for biological function of large-scale gene or protein list [10]. In the present study, Gene Ontology enrichment and KEGG (Kyoto Encyclopedia of Genes and Genomes) pathway analysis of DEGs were conducted using the DAVID online tool. GO analysis included categories of biological processes (BP), cellular component (CC), and molecular function (MF). Pathway analysis is a functional analysis that maps genes to KEGG pathways. And gene count $>2$ and $\mathrm{p}<0.05$ were set as the cutoff point.

2.4. Integration of Protein-Protein Interaction (PPI) Network Analysis. STRING (https://string-db.org/cgi/input.pl) is an online database resource search tool for the retrieval of interacting genes, which include physical and functional associations [11]. In this paper, the STRING online tool was used to construct a protein-protein interaction (PPI) network of upregulation and downregulation DEGs, with a confidence score $>0.4$ defined as significant. Then we imported the interaction data into the Cytoscape software [12] to map a PPI network. Based on the above data, we used Molecular Complex Detection (MCODE) [13], a built-in APP in Cytoscape software, to analyze the interaction relationship of the DEGs encoding proteins and screening hub gene. The parameters of network scoring and cluster finding were set as follows: degree cutoff $=2$, node score cutoff $=0.2$, $\mathrm{k}$-core $=2$, and $\max$ depth $=100$.

2.5. qRT-PCR Validation and Statistical Analysis. Quantitative reverse transcription-PCR was used to validate the hub genes. Total RNA was reverse-transcribed to cDNA using PrimeScript RT reagent Kit with gDNA Eraser (TaKaRa, Japan) according to the manufacturer's instructions. Primer 5.0 software (PREMIER Biosoft, Palo Alto, CA, USA) was used to design primers, and a QuantStudio ${ }^{\mathrm{TM}} 7$ Flex real-time PCR system (Applied Biosystems, Carlsbad, CA, USA) was used. Primers for mRNA are listed in Table 1. All samples were normalized to GAPDH. And the relative expression levels of each gene were calculated using $2-\Delta \Delta \mathrm{Ct}$ methods. Statistical analysis was performed with SPSS software (version 18.0 SPSS Inc.). $\mathrm{P}$ values $<0.05$ were considered statistically significant.

2.6. Patients and Controls. Our study was approved by the ethics committee of the Second Hospital of Jilin University, Jilin University, Jilin, China. 10 healthy donors and 10 knee osteoarthritis patients with knee osteoarthritis (diagnosed according to the ACR classification criteria for knee osteoarthritis) [14] were enrolled, and all gave informed consent. Osteoarthritis synovial membrane samples were 
TABLE 2: The significant enriched analysis of DEGs in osteoarthritis.

\begin{tabular}{|c|c|c|c|c|c|}
\hline Expression & Category & Term & Description & Gene Count & P-Value \\
\hline \multirow{15}{*}{$\begin{array}{l}\text { DOWN- } \\
\text { DEGs }\end{array}$} & $\mathrm{BP}$ & GO:0006911 & phagocytosis, engulfment & 5 & 3.11E-05 \\
\hline & $\mathrm{BP}$ & GO:0045087 & innate immune response & 9 & 0.001731552 \\
\hline & $\mathrm{BP}$ & GO:0043406 & positive regulation of MAP kinase activity & 4 & 0.003542436 \\
\hline & $\mathrm{BP}$ & GO:0006508 & proteolysis & 9 & 0.004361459 \\
\hline & BP & GO:0006956 & complement activation & 4 & 0.010412249 \\
\hline & $\mathrm{CC}$ & GO:0005615 & extracellular space & 24 & 8.12E-08 \\
\hline & $\mathrm{CC}$ & GO:0005576 & extracellular region & 23 & $6.94 \mathrm{E}-06$ \\
\hline & $\mathrm{CC}$ & GO:0042571 & immunoglobulin complex, circulating & 3 & 0.003820636 \\
\hline & $\mathrm{CC}$ & GO:0005886 & plasma membrane & 32 & 0.005732121 \\
\hline & $\mathrm{CC}$ & GO:0000139 & Golgi membrane & 9 & 0.008249883 \\
\hline & MF & GO:0004252 & serine-type endopeptidase activity & 7 & 0.001703259 \\
\hline & MF & GO:0008144 & drug binding & 4 & 0.006434471 \\
\hline & MF & GO:0034987 & immunoglobulin receptor binding & 3 & 0.007358675 \\
\hline & MF & GO:0008201 & heparin binding & 5 & 0.008337436 \\
\hline & MF & GO:0008083 & growth factor activity & 5 & 0.008701217 \\
\hline \multirow{15}{*}{ UP-DEGs } & $\mathrm{BP}$ & GO:0044344 & $\begin{array}{l}\text { cellular response to fibroblast growth } \\
\text { factor stimulus }\end{array}$ & 7 & $2.20 \mathrm{E}-07$ \\
\hline & $\mathrm{BP}$ & GO:0045944 & $\begin{array}{l}\text { positive regulation of transcription from } \\
\text { RNA polymerase II promoter }\end{array}$ & 27 & 4.77E-07 \\
\hline & $\mathrm{BP}$ & GO:0051591 & response to cAMP & 7 & $3.08 \mathrm{E}-06$ \\
\hline & $\mathrm{BP}$ & GO:0000122 & $\begin{array}{l}\text { negative regulation of transcription from } \\
\text { RNA polymerase II promoter }\end{array}$ & 21 & $5.86 \mathrm{E}-06$ \\
\hline & $\mathrm{BP}$ & GO:0043066 & negative regulation of apoptotic process & 16 & $1.31 \mathrm{E}-05$ \\
\hline & $\mathrm{CC}$ & GO:0005634 & nucleus & 75 & $6.25 \mathrm{E}-07$ \\
\hline & $\mathrm{CC}$ & GO:0005654 & nucleoplasm & 47 & 2.11E-06 \\
\hline & $\mathrm{CC}$ & GO:0005737 & cytoplasm & 61 & 0.002569078 \\
\hline & $\mathrm{CC}$ & GO:0005829 & cytosol & 43 & 0.002630227 \\
\hline & $\mathrm{CC}$ & GO:0005667 & transcription factor complex & 6 & 0.02296627 \\
\hline & MF & GO:0001077 & $\begin{array}{l}\text { transcriptional activator activity, RNA } \\
\text { polymerase II core promoter proximal } \\
\text { region sequence-specific binding }\end{array}$ & 12 & $1.03 \mathrm{E}-05$ \\
\hline & MF & GO:0005515 & protein binding & 106 & $2.10 \mathrm{E}-05$ \\
\hline & MF & GO:0000982 & $\begin{array}{l}\text { transcription factor activity, RNA } \\
\text { polymerase II core promoter proximal } \\
\text { region sequence-specific binding }\end{array}$ & 5 & $5.02 \mathrm{E}-05$ \\
\hline & MF & GO:0044822 & poly(A) RNA binding & 25 & 7.91E-05 \\
\hline & MF & GO:0017017 & $\begin{array}{l}\text { MAP kinase tyrosine/serine/threonine } \\
\text { phosphatase activity }\end{array}$ & 4 & $1.95 \mathrm{E}-04$ \\
\hline
\end{tabular}

obtained from OA patients upon total knee replacement at the Second Hospital of Jilin University, and normal synovial membrane samples were obtained from traumatic joint injury cases upon joint synovectomy at the Second Hospital of Jilin University.

\section{Results}

3.1. Identification of DEGs in Osteoarthritis. A total of 20 osteoarthritis synovial membranes and 19 matched normal synovial membranes were analyzed; taking $\mathrm{p}<0.05$ and $|\log \mathrm{FC}|>1$ as a threshold, we extracted 1834 and 1948
DEGs from the expression profile datasets GSE12021 and GSE55235, respectively. By integrated analysis, a total of 258 DEGs were identified, including 161 upregulated DEGs and 97 downregulated DEGs in osteoarthritis samples compared with normal samples.

3.2. GO Functional Enrichment Analysis. To acquire the functions of differential genes, GO function enrichment was analyzed by DAVID online tool, and the DEGs functions were classified into three groups as follows: BP, CC, and MF (Figure 1). As shown in the Figure 1 and Table 2, in the biological processes group, the down-DEGs are mainly enriched in 


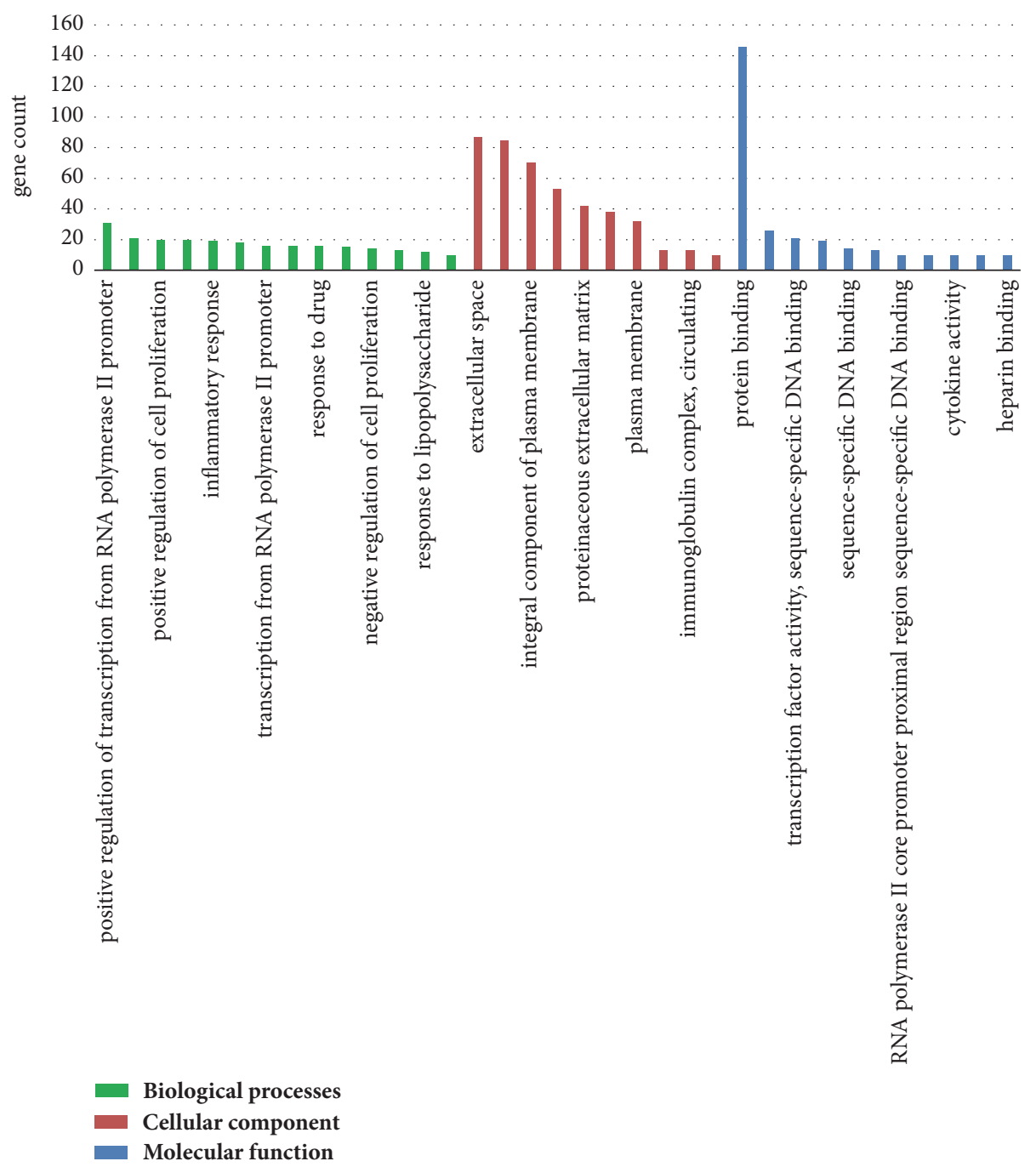

FIGURE 1: Gene Ontology analysis classified the DEGs into 3 groups: molecular function, biological process, and cellular component.

phagocytosis, engulfment, innate immune response, positive regulation of MAP kinase activity, proteolysis, and complement activation, and the up-DEGs are mainly enriched in cellular response to fibroblast growth factor stimulus, response to CAMP, and negative regulation of apoptotic process. In the cellular component group, the down-DEGs are mainly enriched in extracellular space, extracellular region, immunoglobulin complex, and circulating, and the up-DEGs are mainly enriched in nucleus, nucleoplasm, cytoplasm, and cytosol. And in the molecular function group, the downDEGs are mainly enriched in drug binding, immunoglobulin receptor binding, and growth factor activity, and the upDEGs are mainly enriched in transcriptional activator activity, protein binding, poly(A) RNA binding, and MAP kinase tyrosine/serine/threonine phosphatase activity.

3.3. Signaling Pathway Analysis. After the pathway enrichment analysis, downregulated genes were mainly enriched in cytokine-cytokine receptor interaction and glycosphingolipid biosynthesis-globoseries. And upregulated genes were mainly enriched in TNF signaling pathway, osteoclast differentiation, MAPK signaling pathway, NF-kappa B signaling pathway, and rheumatoid arthritis (Figure 2, Table 3).

3.4. PPI Network and Modular Analysis. Based on the data in the STRING database, we constructed a PPI network through Cytoscape software, containing 155 nodes and 625 edges (Figure 3). Among the 155 genes, the top 10 hub genes were identified according to connectivity, including VEGFA, IL6, JUN, IL-1 $\beta$, MYC, IL4, PTGS2, ATF3, EGR1, and DUSP1. IL6 and VEGFA showed the highest degree (degree $=51$ ). In order to further analyze the interaction of protein, 5 modules were detected using the Cytoscape plugin MCODE; the top thee module with score $>5$ were shown in Figure 4. In addition, functional enrichment analyses for these modules were performed. Pathway enrichment analysis showed that Module 1 is mainly associated with TNF signaling pathway, cytokine-cytokine receptor interaction, and osteoclast differentiation. Module 2 is mainly associated with osteoclast differentiation, TNF signaling pathway, and 


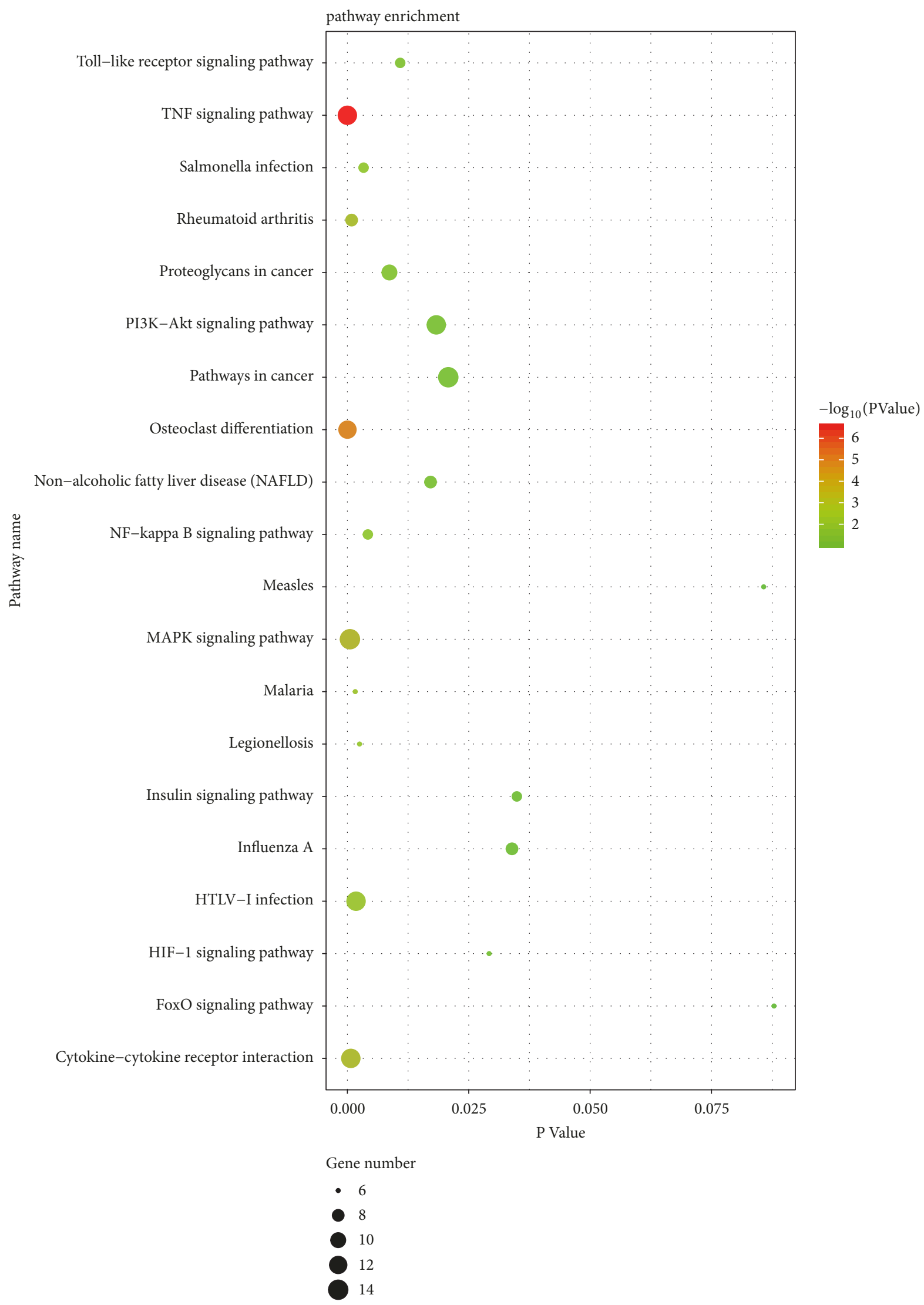

FIGURE 2: Kyoto Encyclopedia of Genes and Genomes (KEGG) enrichment analysis of the pathways. The gradual color represents the P value; the size of the black spots represents the gene number. 
TABLE 3: Signaling pathway enrichment analysis of DEGs function in osteoarthritis.

\begin{tabular}{lcccc}
\hline Expression & Term & Description & Gene Count & P-Value \\
\hline \multirow{2}{*}{ DOWN- DEGs } & hsa04060 & Cytokine-cytokine receptor interaction & 5 & 0.036430433 \\
& hsa00603 & Glycosphingolipid biosynthesis - globoseries & 2 & 0.074364973 \\
\hline & hsa04668 & TNF signaling pathway & 13 & $3.53 \mathrm{E}-09$ \\
& hsa04380 & Osteoclast differentiation & 9 & $1.61 \mathrm{E}-04$ \\
& hsa04010 & MAPK signaling pathway & 12 & $2.13 \mathrm{E}-04$ \\
& hsa05134 & Legionellosis & 6 & $4.41 \mathrm{E}-04$ \\
hsa05132 & Salmonella infection & $5.52 \mathrm{E}-04$ \\
hsa05219 & Bladder cancer & 5 & 0.001397716 \\
& hsa05144 & Malaria & 10 & 0.002719238 \\
& hsa05166 & HTLV-I infection & 6 & 0.003418411 \\
& hsa04064 & NF-kappa B signaling pathway & 6 & 0.003787905 \\
& hsa05323 & Rheumatoid arthritis & 0.003978832 \\
\hline
\end{tabular}

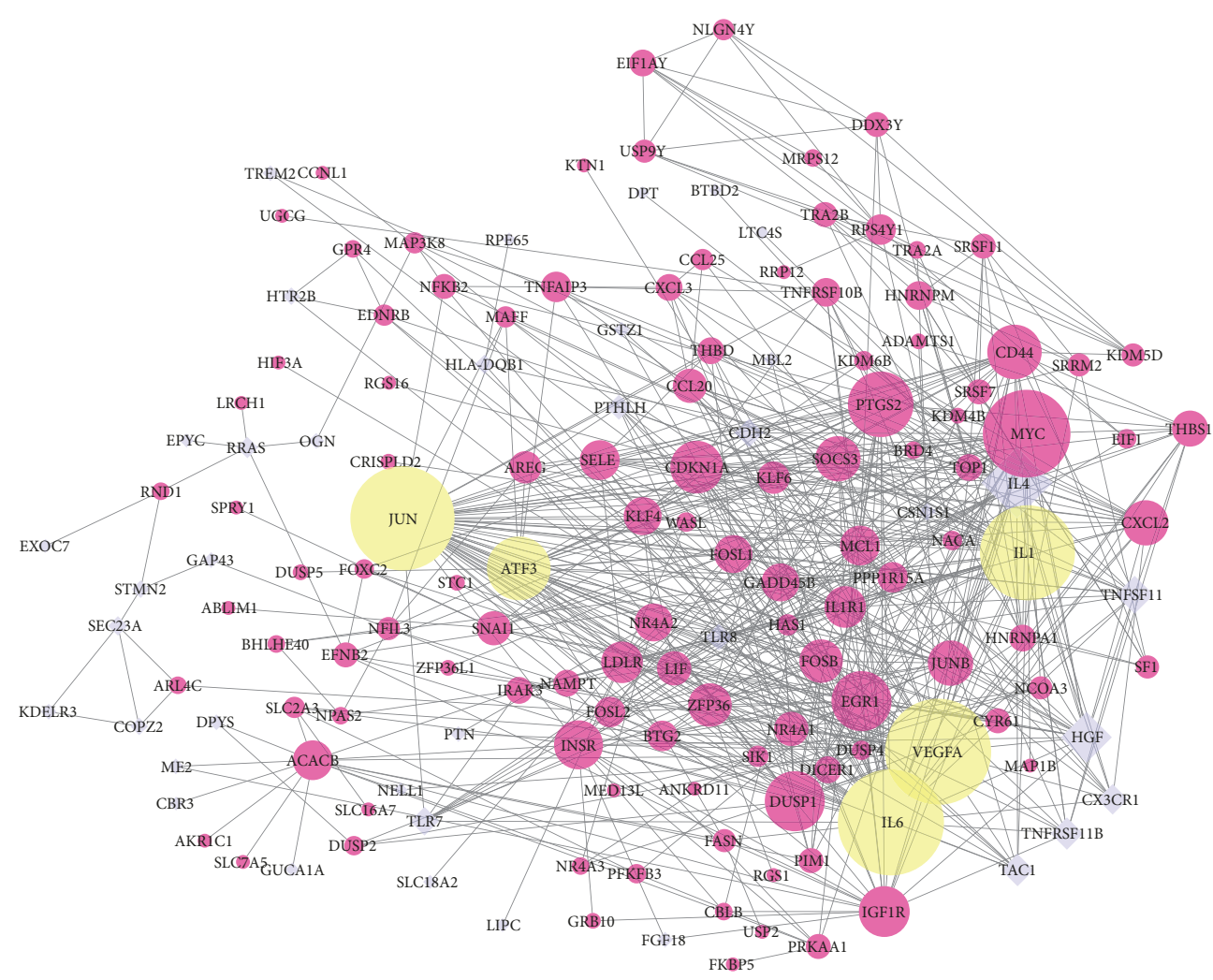

FIGURE 3: PPI network constructed with the upregulated and downregulated DEGs. Red nodes represent upregulated genes, purple nodes represent downregulated genes, and yellow nodes represent upregulated genes validated by qRT-PCR.

cytokine-cytokine receptor interaction. Module 3 is mainly associated with Spliceosome.

3.5. Validation of Hub Gene. To validate microarray results, the expression levels of top 10 hub genes were determined in synovial membrane samples of knee osteoarthritis and normal controls using qRT-PCR. The verification result showed that the expression levels of IL6, VEGFA, JUN, IL-1 $\beta$, and ATF3 were significantly increased in osteoarthritis samples $(\mathrm{p}<0.05)$ (Figure 5). All validations are consistent with the analytical results in this study.

\section{Discussion}

$\mathrm{OA}$ is the most common degenerative joint disease observed worldwide. The prevalence of clinical osteoarthritis has grown to nearly 27 million in the USA [15], and it is a great burden on people's health and medical insurance; therefore, early diagnosis and treatment of osteoarthritis are especially important. Epidemiological studies have demonstrated that osteoarthritis is a multifactorial polygenic disease with numerous environmental and genetic risk factors [16]. It is important to study the molecular mechanisms of the OA. The 

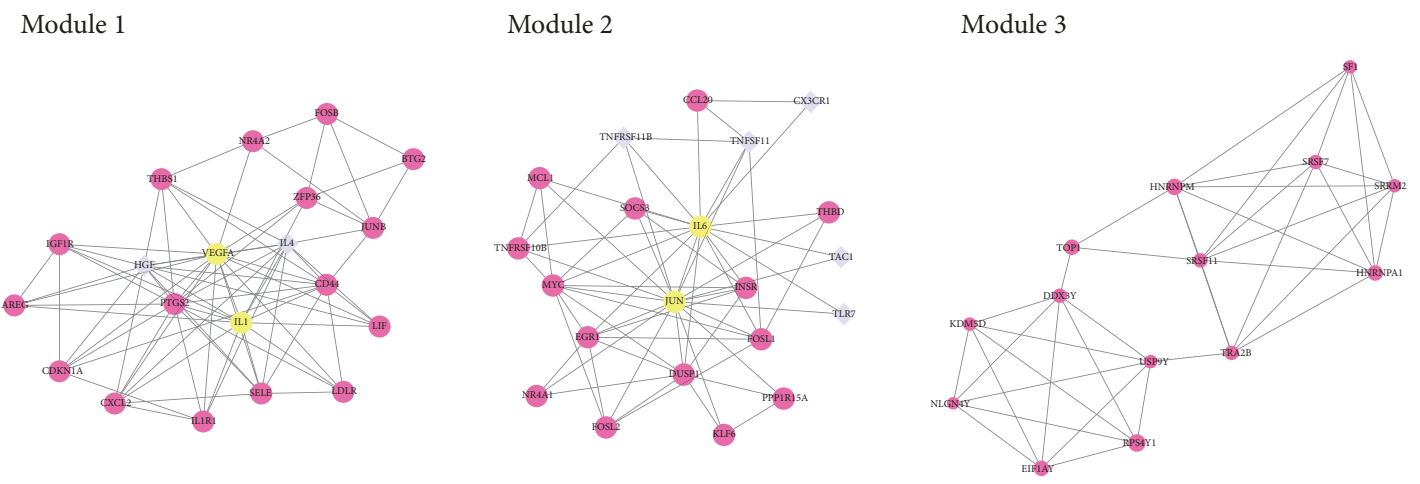

FIGURE 4: The three most significant modules. Red nodes represent upregulated genes, purple nodes represent downregulated genes, and yellow nodes represent upregulated genes validated by qRT-PCR.

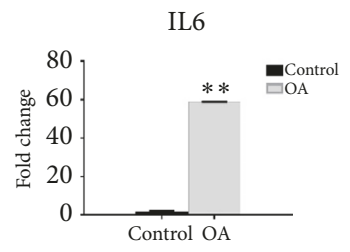

PTGS2

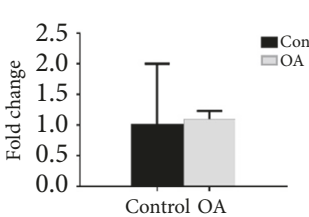

VEGFA

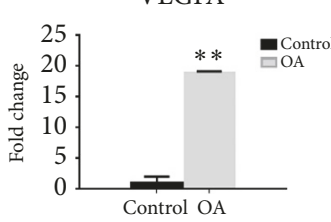

DUSP1

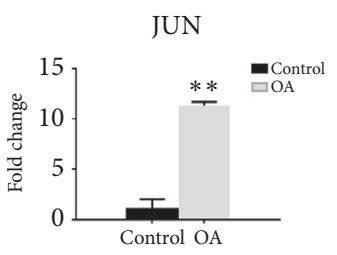

MYC

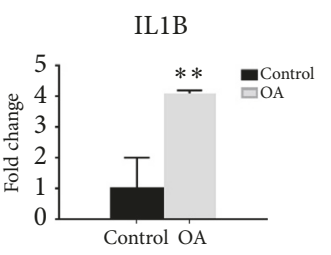

EGR1

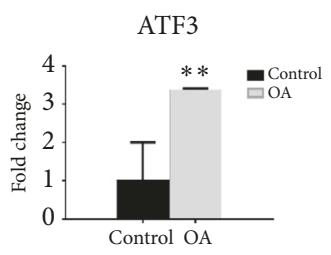

IL4
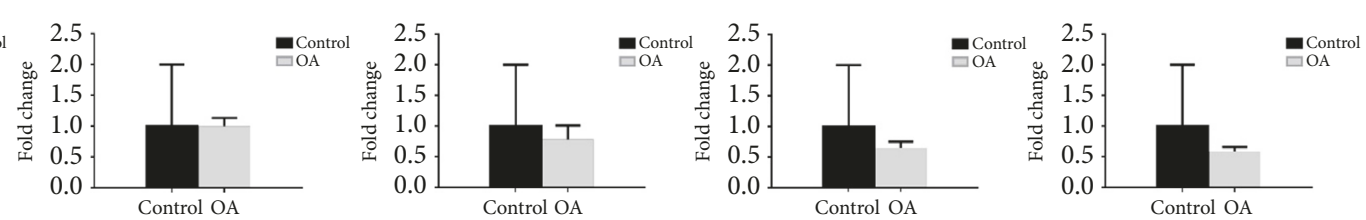

Figure 5: Validation of the top 10 hub genes by qRT-PCR between the OA group $(\mathrm{n}=10)$ and the control group $(\mathrm{n}=10)$. All samples were normalized to the expression of GAPDH, and the relative expression levels of each gene were analyzed using the $2-\Delta \Delta \mathrm{Ct}$ method. ${ }^{* *} \mathrm{P}<0.01$.

previous study on the pathophysiology of osteoarthritis has focused on cartilage and periarticular bone and neglecting the role of synovial tissue in the pathogenesis of osteoarthritis. During OA progression, the synovial membrane is also a source of proinflammatory and catabolic products, and there are multiple pathways and mediators that can directly influence the development and persistence of synovitis [17].

Microarray and high-throughput sequencing technologies have been widely used to predict potential targets gene for osteoarthritis, but most studies focus on a single cohort study or single genetic event. This study integrated two cohorts profile datasets from different groups, and both samples are synovial membranes isolated from knee osteoarthritis patients. Bioinformatics methods are applied to analyze the raw data, and we identify 258 DEGs, including 161 upregulated DEGs and 97 downregulated DEGs. Next, the 258 EDGs were classified into three groups by GO terms using multiple approaches and further clustered based on functions and signaling pathways, respectively.

The DEGs in osteoarthritis analyzed by GO functional enrichment analysis showed that the downregulated DEGs were mainly enriched in immune response, proteolysis, positive regulation of MAP kinase activity, and growth factor activity, while upregulated DEGs were shown to be concerned with cellular response to fibroblast growth factor stimulus, response to cAMP, negative regulation of apoptotic process, and MAP kinase tyrosine. This conforms to our knowledge that immune response, inflammatory responses, and response to cAMP are main mechanisms of OA development and progression [18-22]. According to the previous studies, the participation of the immune system in the development and progression of OA is one of the key elements in the pathogenesis of the disease [23]. It should be noted that the pathophysiological processes occurring in OA are largely mediated by inflammatory cytokines and other anti-inflammatory cytokines that may modulate an inflammatory response and act protectively on joint tissue [24]. The main representatives of anti-inflammatory cytokines involved in the pathogenesis of OA are IL-4, IL-10, and IL-13. In our study, the downregulated gene IL-4 is enriched in immune response, and the dysregulated IL-4 may be involved in the pathogenesis of OA. Furthermore, the enriched KEGG pathways of DEGs and modules analysis included the TNF signaling pathway, MAPK signaling pathway, osteoclast differentiation, and cytokine-cytokine receptor interaction. Previous studies showed that these pathways are involved in osteoarthritis cartilage degeneration and synovial hyperplasia [25-28]. The PPI network was constructed with DEGs, and the top 10 hub genes were as follows: VEGFA, IL6, JUN, IL-1 $\beta$, MYC, IL4, PTGS2, ATF3, EGR1, and DUSP1. The results validated by 
qRT-PCR show that the expression levels of IL6, VEGFA, JUN, IL-1 $\beta$, and ATF3 were significantly increased in OA samples $(\mathrm{p}<0.05)$.

Module analysis of the PPI network suggested that TNF signaling pathway, cytokine-cytokine receptor interaction, and osteoclast differentiation might be involved in OA development. Tumor necrosis factor (TNF) is a critical cytokine, which can induce a wide range of intracellular signal pathways including apoptosis and cell survival as well as inflammation and immunity [29]. TNF- $\alpha$ mediated activation of $\mathrm{NF}-\kappa \mathrm{B}$ signaling pathway is known to play an important role in the pathogenesis of OA [30], and OA was effectively treated by VIP via inhibiting the NF- $\kappa$ B signaling pathway [31]. Cytokines which are produced in joint tissues regulate a broad range of inflammatory processes [32]; the occurrence and development of $\mathrm{OA}$ are driven by various mediators, of which the key role is attributed to the interactions within the cytokine-cytokine network. The inflammatory and antiinflammatory cytokines play a key role in the pathogenesis of OA [33]. Proinflammatory cytokines such as TNF-a, IL$1 \beta$, and IL-17 can enhance osteoclast formation [34]. The role of osteoclasts in chronic arthritis has emerged in recent years; osteoclasts may be key players in the erosive and inflammatory events leading to joint destruction and bone resorption [35].

According to the recent report, the immune system is one of the key elements in the pathogenesis of the OA [23]. Inflammatory cytokines, including IL- $1 \beta, \mathrm{TNF} \alpha$, IL- 6 , and IL-18, play an important role in the development and progression of disease [24]. In our study, inflammatory cytokines IL6 and IL- $1 \beta$ were significantly increased in OA samples. IL$1 \beta$ is one of the representatives of the IL- 1 family; the protein encoding by IL- $1 \beta$ induces inflammatory reactions and catabolic effect. The level of IL- $1 \beta$ has elevated in the synovial membrane, synovial fluid, cartilage, and the subchondral bone $[36,37]$. The biological activation of synovial cells by IL- $1 \beta$ is mediated by the interaction between IL-1R 1 and IL-1R2 receptor; blocking their connection with IL- $1 \beta$ may decrease in the activity of IL- $1 \beta$ [38]. Downregulation of the production and activity of active proinflammatory and procatabolic IL-1 $\beta$ is optimal for OA molecular therapy [39]. IL6 gene encodes a cytokine that strongly activates the immune system and inflammatory response; the protein is primarily produced at sites of acute and chronic inflammation, where it is secreted into the serum and induces a transcriptional inflammatory response through interleukin 6 receptor, alpha. The production of IL- 6 in the degenerative joint is usually in response to IL-1 $\beta$ and TNF $\alpha$ and is mainly implemented by chondrocytes and osteoblasts [40], cooperating with IL-1 $\beta$ and $\mathrm{TNF} \alpha$, activation of osteoclasts formation, and thus bone resorption [41]. In synergy with another cytokine, IL6 causes an increase in the production of enzymes and decrease in type II collagen [42].

VEGFA is the founding member of the VEGF family and is the most widely studied gene in the molecular mechanism of OA. VEGFA gene encodes a heparin-binding protein, which induces proliferation and migration of vascular endothelial cells, and is essential for both physiological and pathological angiogenesis. VEGF is an important mediator of bone development [43]. Increased VEGF levels are associated with OA progression, and it is involved in pathologies including synovitis, cartilage degeneration, osteophyte formation, and pain [44]. During the advanced stage of OA, VEGF expression has been found increased in the articular cartilage and synovium [45]. Synergy with IL-1 $\beta$, VEGF was found to significantly reduce the expression of aggrecan and type II collagen at the gene and protein levels [46]. VEGF expression increased in synovial macrophages and fibroblastlike synovial cells, and the expression of TNF- $\alpha$ and IL- 6 [47] increased as well. There is growing evidence suggesting the pathological involvement of VEGF and its signaling pathways. Treatments targeting VEGF signaling will be a supplement of traditional treatments in OA.

The involvement of c-Jun N-terminal kinase (JNK) in signaling transduction pathways has been well-characterized in articular chondrocytes [48]. The basic leucine zipper transcription factor, ATF-like (BATF), a member of the Activator protein-1 family (AP-1), promotes transcriptional activation or repression, depending on the interacting partners (JUNB or C-JUN), BATF, which forms a heterodimeric complex with JUN-B, and C-JUN may play important roles in OA cartilage destruction through regulating anabolic and catabolic gene expression in chondrocytes [49]. The involvement of ATF3 in joint disease has not been well studied, the ATF3 gene which belongs to the ATF/cAMP-responsive elementbinding protein family and encodes a member of the activating transcription factor [50]. ATF3 expression significantly increased in the OA cartilage, and ATF3 deficiency decreased cytokine-induced IL6 transcription in chondrocytes through repressing NF-kB signaling. The deficiency of ATF3 may alleviate articulfvar degeneration of OA patient; ATF3 and its related pathways may be a suitable drug target for the treatment of OA $[51,52]$.

In summary, by means of data processing and qRT-PCR validation, the hub genes including IL6, VEGFA, JUN, IL-1 $\beta$, and ATF3 may have the potential to be used as drug targets and diagnostic markers of OA. Although several hub genes and pathways were identified and validated in our study, there were still some limitations: small sample size was used for the analyses and there was lack of further experiment. Further experimental studies with larger sample size are needed to confirm our analysis result.

\section{Abbreviations}

OA: Osteoarthritis

DEGs: Differentially expressed genes

GEO: Gene Expression Omnibus

PPI: $\quad$ Protein-protein interaction

GO: $\quad$ Gene Ontology

KEGG: Kyoto Encyclopedia of Genes and Genomes

GWAS: Genomewide association studies

BP: Biological processes

CC: Cellular component

MF: $\quad$ Molecular function

MCODE: Molecular Complex Detection

VEGFA: Vascular endothelial growth factor A

IL6: Interleukin 6 
JUN: Jun protooncogene

IL1 $\beta$ : Interleukin 1 beta

MYC: MYC protooncogene

IL4: Interleukin 4

PTGS2: Prostaglandin-endoperoxide synthase 2

ATF3: Activating transcription factor 3

EGR1: Early growth response 1

DUSP1: Dual specificity phosphatase 1

cAMP: Cyclic adenosine monophosphate

cDNA: DNA complementary to RNA

MAPK: Mitogen-activated protein kinase.

\section{Data Availability}

The data used to support the findings of this study are available from the corresponding author upon request.

\section{Conflicts of Interest}

The authors declare that they have no conflicts of interest.

\section{Authors' Contributions}

Guizhen Zhang and Yang Song designed and supervised the study; Zhaoyan Li, Qingyu Wang, Gaoyang Chen, and Xin Li performed the analysis work; Zhenwu Du, Qiwei Yang, and Ming Ren contributed to the data analysis; Guizhen Zhang and Zhaoyan Li organized, designed, and wrote the paper. All authors reviewed the final manuscript.

\section{Acknowledgments}

This work was supported by the project supported by the National Natural Science Foundation of China (Grant no. 81702195), the Department of Science and Technology of Jilin Province, China (Grant no. 20180520125JH), the Education Department of Jilin Province (Grant no. JJKH20180103KJ), the Project of Bethune Youth Foundation of Jilin University, China (Grant no. 2015409), and the Project of Application Demonstration Center of Precision Medicine for Molecular Diagnosis in Jilin Province (2016-2018, NDRC).

\section{References}

[1] K. Sinusas, "Osteoarthritis:Diagnosis and treatment," American Family Physician, vol. 85, no. 1, pp. 49-56, 2012.

[2] J. M. Hootman and C. G. Helmick, "Projections of US prevalence of arthritis and associated activity limitations," Arthritis \& Rheumatism, vol. 54, no. 1, pp. 226-229, 2006.

[3] M. C. Hochberg, L. Yerges-Armstrong, M. Yau, and B. D. Mitchell, "Genetic epidemiology of osteoarthritis: Recent developments and future directions," Current Opinion in Rheumatology, vol. 25, no. 2, pp. 192-197, 2013.

[4] M. S. Yau, L. M. Yerges-Armstrong, Y. Liu et al., "GenomeWide Association Study of Radiographic Knee Osteoarthritis in North American Caucasians," Arthritis Rheumatol, vol. 69, no. 2, pp. 343-351, 2017.

[5] R. Huber, C. Hummert, U. Gausmann et al., "Identification of intra-group, inter-individual, and gene-specific variances in
mRNA expression profiles in the rheumatoid arthritis synovial membrane," Arthritis Research \& Therapy, vol. 10, no. 4, p. R98, 2008.

[6] D. Woetzel, R. Huber, P. Kupfer et al., "Identification of rheumatoid arthritis and osteoarthritis patients by transcriptome-based rule set generation," Arthritis Research \& Therapy, vol. 16, no. 2, article no. R84, 2014.

[7] T. Barrett, S. E. Wilhite, P. Ledoux et al., "NCBI GEO: archive for functional genomics data sets-update," Nucleic Acids Research, vol. 41, no. 1, pp. D991-D995, 2013.

[8] L. Gautier, L. Cope, B. M. Bolstad, and R. A. Irizarry, "Affyanalysis of Affymetrix GeneChip data at the probe level," Bioinformatics, vol. 20, no. 3, pp. 307-315, 2004.

[9] M. E. Ritchie, B. Phipson, D. Wu et al., "limma powers differential expression analyses for RNA-sequencing and microarray studies," Nucleic Acids Research, 2015.

[10] D. W. Huang, B. T. Sherman, Q. Tan et al., "The DAVID gene functional classification tool: a novel biological module-centric algorithm to functionally analyze large gene lists," Genome Biology, vol. 8, no. 9, article R183, 2007.

[11] D. Szklarczyk, A. Franceschini, M. Kuhn et al., "The STRING database in 2011: functional interaction networks of proteins, globally integrated and scored," Nucleic Acids Research, vol. 39, no. 1, pp. D561-D568, 2011.

[12] P. Shannon, A. Markiel, O. Ozier et al., "Cytoscape: a software Environment for integrated models of biomolecular interaction networks," Genome Research, vol. 13, no. 11, pp. 2498-2504, 2003.

[13] G. D. Bader and C. W. V. Hogue, "An automated method for finding molecular complexes in large protein interaction networks," BMC Bioinformatics, vol. 4, no. 1, p. 2, 2003.

[14] G. Peat, E. Thomas, R. Duncan, L. Wood, E. Hay, and P. Croft, "Clinical classification criteria for knee osteoarthritis: Performance in the general population and primary care," Annals of the Rheumatic Diseases, vol. 65, no. 10, pp. 1363-1367, 2006.

[15] R. C. Lawrence, D. T. Felson, C. G. Helmick et al., "Estimates of the prevalence of arthritis and other rheumatic conditions in the United States. Part II," Arthritis \& Rheumatology, vol. 58, no. 1, pp. 26-35, 2008.

[16] E. Zengini, C. Finan, and J. M. Wilkinson, "The genetic epidemiological landscape of hip and knee osteoarthritis: Where are we now and where are we going?" The Journal of Rheumatology, vol. 43, no. 2, pp. 260-266, 2016.

[17] C. R. Scanzello and S. R. Goldring, "The role of synovitis in osteoarthritis pathogenesis," Bone, vol. 51, no. 2, pp. 249-257, 2012.

[18] C. Bui, M. J. Barter, J. L. Scott et al., "cAMP response element-binding (CREB) recruitment following a specific $\mathrm{CpG}$ demethylation leads to the elevated expression of the matrix metalloproteinase 13 in human articular chondrocytes and osteoarthritis," The FASEB Journal, vol. 26, no. 7, pp. 3000-3011, 2012.

[19] A. M. Kandahari, X. Yang, A. S. Dighe, D. Pan, and Q. Cui, "Recognition of Immune Response for the Early Diagnosis and Treatment of Osteoarthritis," Journal of Immunology Research, vol. 2015, Article ID 192415, 13 pages, 2015.

[20] D. L. Long, V. Ulici, S. Chubinskaya, and R. F. Loeser, "Heparinbinding epidermal growth factor-like growth factor (HBEGF) is increased in osteoarthritis and regulates chondrocyte catabolic and anabolic activities," Osteoarthritis and Cartilage, vol. 23, no. 9, pp. 1523-1531, 2015. 
[21] S. Y. McCoy, K. A. Falgowski, P. P. Srinivasan, W. R. Thompson, E. M. Selva, and C. B. Kirn-Safran, "Serum xylosyltransferase 1 level increases during early posttraumatic osteoarthritis in mice with high bone forming potential," Bone, vol. 51, no. 2, pp. 224231, 2012.

[22] B. Xia, Di Chen, J. Zhang, S. Hu, H. Jin, and P. Tong, "Osteoarthritis Pathogenesis: A Review of Molecular Mechanisms," Calcified Tissue International, vol. 95, no. 6, pp. 495-505, 2014.

[23] M. B. Goldring and M. Otero, "Inflammation in osteoarthritis," Current Opinion in Rheumatology, vol. 23, no. 5, pp. 471-478, 2011.

[24] P. Wojdasiewicz, Ł. A. Poniatowski, and D. Szukiewicz, "The role of inflammatory and anti-inflammatory cytokines in the pathogenesis of osteoarthritis," Mediators of Inflammation, vol. 2014, Article ID 561459, 19 pages, 2014.

[25] Y. Lai, X. Bai, Y. Zhao et al., "ADAMTS-7 forms a positive feedback loop with TNF- $\alpha$ in the pathogenesis of osteoarthritis," Annals of the Rheumatic Diseases, vol. 73, pp. 1575-1584, 2014.

[26] J. Lee, C. Park, H. J. Kim et al., "Stimulation of osteoclast migration and bone resorption by $\mathrm{C}-\mathrm{C}$ chemokine ligands 19 and 21," Experimental \& Molecular Medicine, vol. 49, no. 7, p. e358, 2017.

[27] X. Li, J. Yang, D. Liu et al., "Knee loading inhibits osteoclast lineage in a mouse model of osteoarthritis," Scientific Reports, vol. 6 , no. 1, 2016.

[28] Y. Wu, W. Liu, L. Zhang et al., "Effects of microRNA-24 targeting $\mathrm{C}$-myc on apoptosis, proliferation and cytokine expressions in chondrocytes of rats with osteoarthritis via MAPK signaling pathway," Journal of Cellular Biochemistry, 2017.

[29] W.-M. Chu, “Tumor necrosis factor," Cancer Letters, vol. 328, no. 2, pp. 222-225, 2013.

[30] Y. Lai, X. Bai, Y. Zhao et al., "ADAMTS-7 forms a positive feedback loop with TNF- $\alpha$ in the pathogenesis of osteoarthritis," Annals of the Rheumatic Diseases, vol. 2013, 2013.

[31] Y. Liang, S. Chen, Y. Yang et al., "Vasoactive intestinal peptide alleviates osteoarthritis effectively via inhibiting NF- $\kappa$ B signaling pathway," Journal of Biomedical Science, vol. 25, no. 1, 2018.

[32] I. B. McInnes and G. Schett, "Cytokines in the pathogenesis of rheumatoid arthritis," Nature Reviews Immunology, vol. 7, no. 6, pp. 429-442, 2007.

[33] J. C. Fernandes, J. Martel-Pelletier, and J. Pelletier, "The role of cytokines in osteoarthritis pathophysiology," Biorheology, vol. 39, no. 1-2, pp. 237-246, 2002.

[34] K. Redlich, S. Hayer, R. Ricci et al., "Osteoclasts are essential for TNF- $\alpha$-mediated joint destruction," The Journal of Clinical Investigation, vol. 110, no. 10, pp. 1419-1427, 2002.

[35] N. Maruotti, M. Grano, S. Colucci, F. D’Onofrio, and F. P. Cantatore, "Osteoclastogenesis and arthritis," Clinical and Experimental Medicine, vol. 11, no. 3, pp. 137-145, 2011.

[36] C. Melchiorri, R. Meliconi, L. Frizziero et al., "Enhanced and coordinated in vivo expression of inflammatory cytokines and nitric oxide synthase by chondrocytes from patients with osteoarthritis," Arthritis \& Rheumatology, vol. 41, no. 12, pp. 2165-2174, 1998.

[37] F. Massicotte, D. Lajeunesse, M. Benderdour et al., "Can altered production of interleukin- $\beta$, interleukin- 6 , transforming growth factor- $\beta$ and prostaglandin E2 by isolated human subchondral osteoblasts identity two subgroups of osteoarthritic patients," Osteoarthritis and Cartilage, vol. 10, no. 6, pp. 491-500, 2002.
[38] G. Palmer, P.-A. Guerne, F. Mezin et al., "Production of interleukin-1 receptor antagonist by human articular chondrocytes," Arthritis Research \& Therapy, vol. 4, no. 3, pp. 226-231, 2002.

[39] Z. Jotanovic, R. Mihelic, B. Sestan, and Z. Dembic, "Role of interleukin-1 inhibitors in osteoarthritis: An evidence-based review," Drugs \& Aging, vol. 29, no. 5, pp. 343-358, 2012.

[40] S. Bender, H.-D. Haubeck, E. Van de Leur et al., "Interleukin$1 \beta$ induces synthesis and secretion of interleukin- 6 in human chondrocytes," FEBS Letters, vol. 263, no. 2, pp. 321-324, 1990.

[41] K. T. Steeve, P. Marc, T. Sandrine, H. Dominique, and F. Yannick, "IL-6, RANKL, TNF-alpha/IL-1: interrelations in bone resorption pathophysiology," Cytokine \& Growth Factor Reviews, vol. 15, no. 1, pp. 49-60, 2004.

[42] B. Porée, M. Kypriotou, C. Chadjichristos et al., "Interleukin-6 (IL-6) and/or soluble IL-6 receptor down-regulation of human type II collagen gene expression in articular chondrocytes requires a decrease of Sp1.Sp3 ratio and of the binding activity of both factors to the COL2A1 promoter," The Journal of Biological Chemistry, vol. 283, no. 8, pp. 4850-4865, 2008.

[43] E. Zelzer and B. R. Olsen, "Multiple Roles of Vascular Endothelial Growth Factor (VEGF) in Skeletal Development, Growth, and Repair," vol. 65 of Current Topics in Developmental Biology, pp. 169-187, Elsevier, 2004.

[44] J. L. Hamilton, M. Nagao, B. R. Levine, D. Chen, B. R. Olsen, and H.-J. Im, "Targeting VEGF and Its Receptors for the Treatment of Osteoarthritis and Associated Pain," Journal of Bone and Mineral Research, vol. 31, no. 5, pp. 911-924, 2016.

[45] J. R. Jackson, J. A. Minton, H. o. ML, N. Wei, and J. D. Winkler, "Expression of vascular endothelial growth factor in synovial fibroblasts is induced by hypoxia and interleukin lbeta," The Journal of Rheumatology, vol. 24, no. 7, pp. 1253-1259, 1997.

[46] X.-Y. Chen, Y.-R. Hao, Z. Wang, J.-L. Zhou, Q.-X. Jia, and B. Qiu, "The effect of vascular endothelial growth factor on aggrecan and type II collagen expression in rat articular chondrocytes," Rheumatology International, vol. 32, no. 11, pp. 3359-3364, 2012.

[47] X. Duan, Q. Li, L. J. Lin et al., "Expression of hypoxiainducible factor-1a and vascular endothelial growth factor in the synovium of patients with osteoarthritis," Nan Fang Yi Ke Da Xue Xue Bao, vol. 31, no. 1, pp. 117-120, 2011.

[48] S. Islam, T. Kermode, D. Sultana et al., "Expression profile of protein tyrosine kinase genes in human osteoarthritis chondrocytes," Osteoarthritis and Cartilage, vol. 9, no. 8, pp. 684-693, 2001.

[49] J. Rhee, S.-H. Park, S.-K. Kim et al., "Inhibition of BATF/JUN transcriptional activity protects against osteoarthritic cartilage destruction," Annals of the Rheumatic Diseases, vol. 76, no. 2, pp. 427-434, 2017.

[50] G. Liang, C. D. Wolfgang, B. P. C. Chen, T.-H. Chen, and T. Hai, "ATF3 gene: Genomic organization, promoter, and regulation," The Journal of Biological Chemistry, vol. 271, no. 3, pp. 1695-1701, 1996.

[51] T. Iezaki, K. Ozaki, K. Fukasawa et al., "ATF3 deficiency in chondrocytes alleviates osteoarthritis development," The Journal of Pathology, vol. 239, no. 4, pp. 426-437, 2016.

[52] E. Kim, H. Y. Shin, J. Kim et al., "ATF3 Plays a Key Role in Kdo2Lipid A-Induced TLR4-Dependent Gene Expression via NF- $\kappa \mathrm{B}$ Activation," PLoS ONE, vol. 5, no. 12, p. e14181, 2010. 


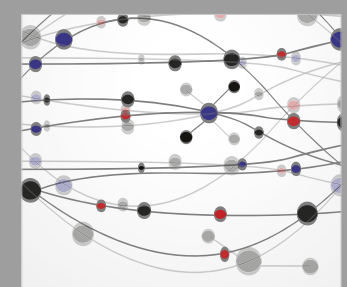

The Scientific World Journal
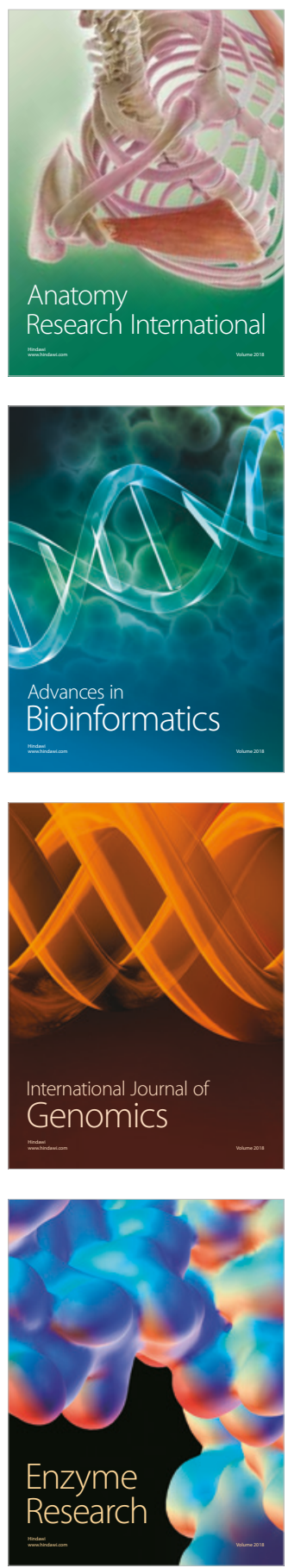
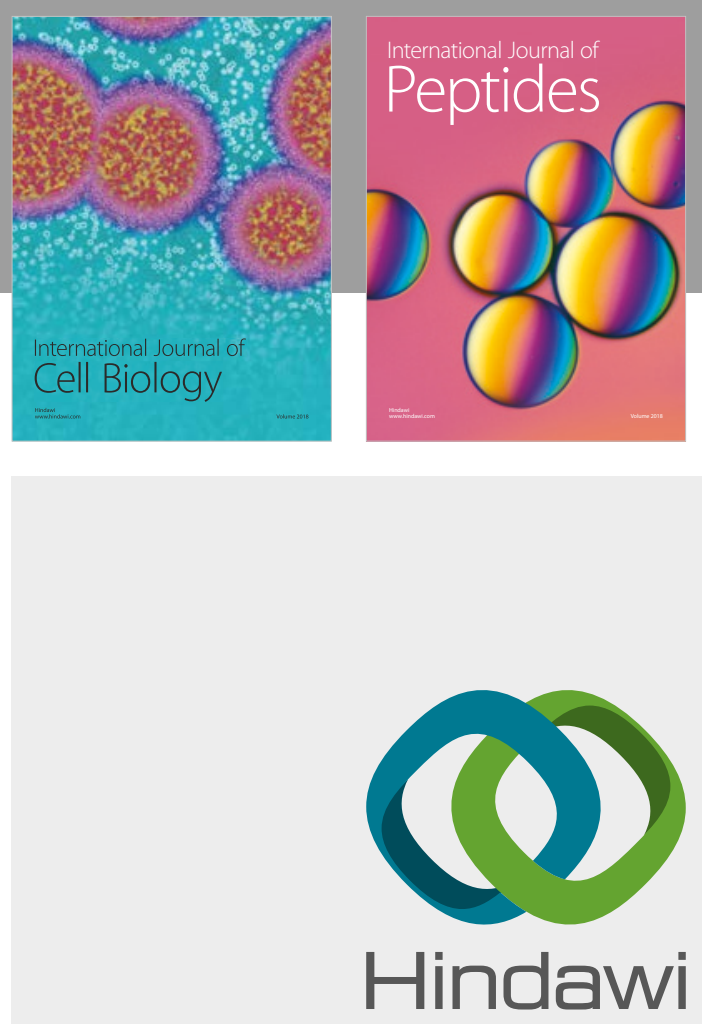

Submit your manuscripts at

www.hindawi.com
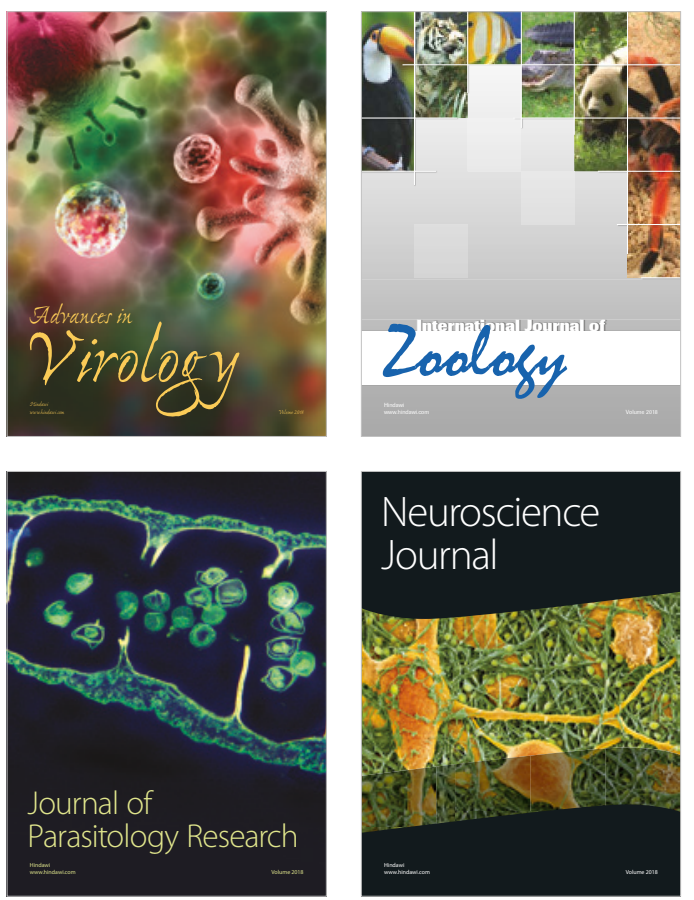
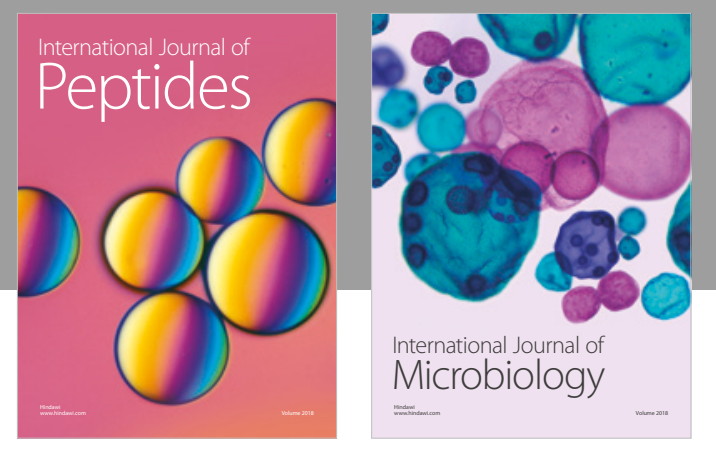

nternational Journal of Microbiology
Journal of
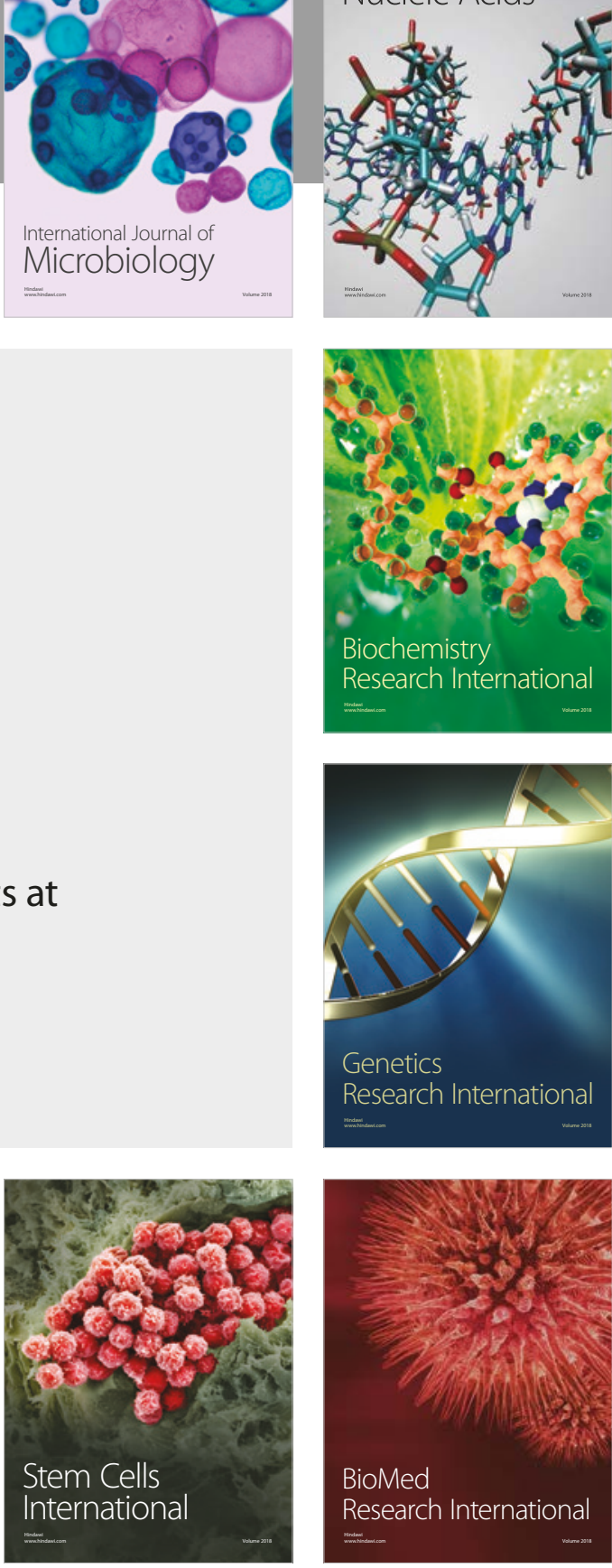
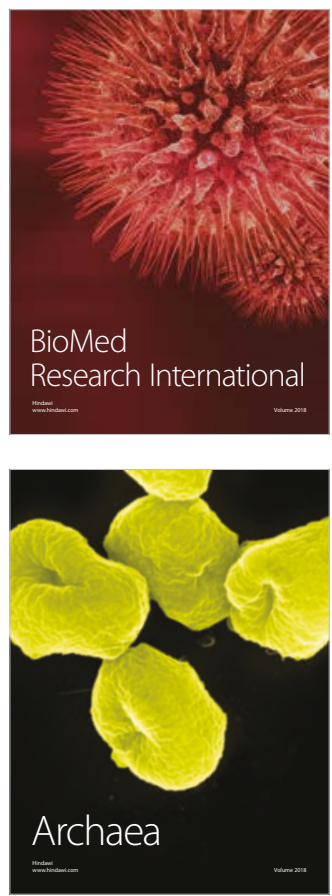